\title{
Maturation and substrate processing topography of the Plasmodium falciparum invasion/egress protease plasmepsin $\mathrm{X}$
}

Daniel Goldberg ( $\sim$ dgoldberg@wustl.edu )

Washington University in St. Louis https://orcid.org/0000-0003-3529-8399

Sumit Mukherjee

Washington University

Eashan Sharma

Washington University

Article

Keywords:

Posted Date: January 7th, 2022

DOI: https://doi.org/10.21203/rs.3.rs-1214907/v1

License: (c) (i) This work is licensed under a Creative Commons Attribution 4.0 International License.

Read Full License

Version of Record: A version of this preprint was published at Nature Communications on August 4th, 2022. See the published version at https://doi.org/10.1038/s41467-022-32271-7. 


\section{Abstract}

During the intravascular stage of infection, the malaria parasite Plasmodium invades a host erythrocyte, multiplies within a parasitophorous vacuole (PV) and exits upon rupture of the PV and erythrocyte membranes in a process known as egress. Both egress and invasion are controlled by effector proteins discharged from specialized secretory organelles. The aspartic protease plasmepsin X (PM X) regulates activity for many of these effectors, but it is unclear how PM X accesses its diverse substrates that reside in different organelles. PM X also processes itself to generate different isoforms that remain present in terminal schizonts. The function of these different forms is not understood. We have mapped the autoprocessing cleavage sites and constructed parasites with cleavage site mutations. Surprisingly, all the cleavage mutant forms of PM X, including a quadruple mutant that remained full-length, retained in vitro activity, were trafficked normally in the parasites, and supported parasite growth and normal egress and invasion. Further analysis showed that the $\mathrm{N}$-terminal half of the prodomain stays bound to the catalytic domain even after processing and is required for proper folding and intracellular trafficking of PM X. We find that this enzyme cleaves microneme and exoneme substrates before discharge, possibly in a common precursor organelle, while the rhoptry substrates that are dependent on PM X activity are cleaved after exoneme discharge into the PV. The data give insight into the temporal, spatial and biochemical control of this unusual but important aspartic protease.

\section{Introduction}

Malaria is caused by the parasitic protozoan Plasmodium and accounts for more than 600,000 deaths annually ${ }^{1}$. The majority of deaths are due to infection with Plasmodium falciparum. The disease symptoms result exclusively from the blood stage of infection during which the parasite replicates asexually within the host red blood cells (RBCs) through schizogony. Each schizont matures into 8-32 merozoites and, by rupturing the parasitophorous vacuolar membrane (PVM) surrounding the parasite as well as the red blood cell membrane (RBCM), exit from the host cells by a process called "egress"2. The egressed merozoites subsequently invade fresh RBCs. Given the importance of the intraerythrocytic cycles of egress and invasion in the spread of the infection, it is, therefore, of considerable interest to target these processes for the development of disease intervention strategies.

The Plasmodium aspartic protease plasmepsin $\mathrm{X}(\mathrm{PM} \mathrm{X})$ is essential for replication of $P$. falciparum during the intraerythrocytic cycle $\mathrm{e}^{3-5}$. In mature schizonts, $\mathrm{PM} X$ is located in the exoneme secretory organelles and is involved in the proteolytic activation of the other exoneme-localized protease subtilisinlike protease 1 (SUB1). Upon release into the parasitophorous vacuole (PV), SUB1 encounters and activates downstream effectors such as serine repeat antigens (SERAs) and the merozoite surface proteins (MSPs) ${ }^{6-8}$. Concerted activities of these molecules lead to rupture of both the PVM and the $\mathrm{RBCM}$, releasing the daughter parasites into the blood stream. Importantly, PM X is druggable, as multiple classes of compounds targeting PM X have high efficacies both in vitro and in vivo as well as being active on different life stages ${ }^{3-5}$. 
Recent data suggest that aside from SUB1, PM X has additional substrates in other secretory organelles that function during parasite invasion ${ }^{5}$. Among these substrates, the apical membrane antigen1 (AMA1), subtilisin 2 (SUB2) and the erythrocyte binding antigens (EBAs) are targeted to the micronemes ${ }^{9,10}$, whereas the reticulocyte binding protein homologs (Rhs) are trafficked to the rhoptries ${ }^{11}$. These substrates are therefore spatially segregated from PM X in mature schizonts. Although it is not known how PM X-mediated cleavage affects the intracellular trafficking and functionalities of these effector proteins, clearly the findings indicate that PM X activity inside the parasites is rather complex, and that the protease might be active in more than one subcellular compartment.

Structurally PM X has an N-terminal signal peptide (SP) sequence followed by a long prodomain (PD) and a C-terminal catalytic domain. Initially synthesized as a $64 \mathrm{kDa}$ precursor, it is subsequently converted into multiple shorter forms through autoproteolytic maturation ${ }^{5}$. The processing of PM X never goes to completion, as both unprocessed and processed forms are detected in terminal schizonts ${ }^{3}$. However, the significance of this partial processing remains unknown. Recent data demonstrate a PM Xspecific inhibitor can bind to multiple processed forms of PM X, suggesting that they may be active ${ }^{5}$. Given the differentially localized substrate repertoire along with the presence of multiple processed forms of PM X in the schizonts, the present study sought to address the following important question: how does the processing regulate $\mathrm{PM} X$ trafficking, activity, and substrate specificity in the malaria parasites? We find that PM $X$ has unusual characteristics for an aspartic protease and provide cellular evidence to explain its ability to process substrates in different compartments.

\section{Results}

\subsection{PM X undergoes autocleavage at multiple alternative sites that are independent of each other.}

Previously we reported that multiple processed and unprocessed forms of PM X remain in terminal schizonts ${ }^{3}$. To shed light on the significance of PM X processing, it was of interest to determine the cleavage sites. However, because of low abundance of PM X in parasites, we were unable to extract enough native enzyme from parasite lysates for $\mathrm{N}$-terminal sequence analysis. We therefore took advantage of the recombinant PM X (rPM X) that we could successfully express as a C-terminally $8 \mathrm{X}$ histidine-tagged secreted protein from mammalian cells. The protein was a mixture of differentially processed polypeptides (Fig. 1a). Of these, one polypeptide of approximately $16 \mathrm{kDa}$ molecular weight was detected only in the Coomassie blot but not in the western blot with an anti-His antibody, suggesting that it is an $\mathrm{N}$-terminal fragment (red arrow in Fig. 1a). To precisely determine its composition, we excised the band from the gel and subjected it to proteomic analysis (Supplementary MS file 1). This confirmed that the $16 \mathrm{kDa}$ fragment represents the $\mathrm{N}$-terminal prodomain fragment of rPM X (Fig. 1b, red arrow). Using a similar approach, among the higher molecular weight fragments that retained the C-terminal $8 \mathrm{x}$ His tag, we were able to determine the sequence of the $44 \mathrm{kDa}$ polypeptide (Fig. 1a-b, blue arrows; Supplementary MS file 2). Importantly, the N-terminus of this polypeptide and the C-terminus of the 16 
kDa polypeptide ended at amino acids leucine and phenylalanine respectively, which are non-tryptic sites (open arrow ends in Fig. 1b). This allowed us to map cleavage sites defining semi-pro and mature isoforms of plasmepsin $\mathrm{X}$.

We mutated the identified cleavage site at the pro-mature junction (IALE to IAAA). This di-mutation had been shown to abolish cleavage of a fluorogenic peptide by isolated PM $X^{4,5}$. The mutated version of PM $X$ expressed in mammalian cells exhibited processing indistinguishable from that of wild type (Fig. $1 \mathrm{C}$ ). 7 amino acids upstream of this cleavage site is a tetrapeptide motif that corresponds to the PM X peptide cleavage specificity defined by Favuzza et al. ${ }^{5}$. Mutation of this alternate site alone had no effect, but mutation of both sites together prevented processing to the mature form, while allowing processing to the semi-pro form. Mutation of the intra-prodomain cleavage site yielded a more complex pattern (Fig. 1c, d) and suggested alternative cleavage. A site conforming to the PM X peptide cleavage specificity was identified 20 amino acids downstream (Fig. 1b).

To further analyze these cleavages, we moved to cultured parasites and expressed PM X mutants as second copies. Mutation of both intra-prodomain sites together abolished processing to the semi-pro but not to the mature form (Fig. 1e). Mutation of both cleavage sites at the pro-mature junction abolished processing to the mature form but allowed processing to the semi-pro form. Mutation of all four sites together blocked all processing and resulted in accumulation of proenzyme. The data suggest that similar to the mammalian rPM X, PM X expressed in parasites undergoes autocatalytic processing at multiple alternative sites that are independent of each other.

\subsection{Proteolytic processing is not necessary for parasite growth or for activity of $P M X$.}

Our second copy mutants were made in an endogenous PM X TetR-regulated knockdown line, PM Xapt3. Wild-type second copy PM X but not a catalytically dead mutant (D266G) can rescue the growth defect that is seen when parasites are grown in the absence of anhydrotetracycline (aTc) to deplete endogenous PM X (as seen previously, ref. 3 and Supplementary Fig. 1). Surprisingly, each of the cleavage site mutants including the quadruple mutant (Quad ${ }^{\text {mut }}$ ) was able to rescue growth in the absence of aTc (Fig. 2a). We analyzed the processing of PM X substrate proteins SUB1 and AMA1 by the different cleavage mutant forms of PM $X$ when endogenous PM X was knocked down. As previously reported ${ }^{3}$, withdrawal of aTc in the parental PM X ${ }^{\text {apt }}$ line results in a processing defect in conversion of the SUB1 54 $\mathrm{kDa}$ intermediate to the mature $47 \mathrm{kDa}$ form (Fig. 2b). This PM X knockdown also impairs conversion of the AMA1 70kDa precursor to the $60 \mathrm{kDa}$ form. Processing of both proteins was fully restored by second copy expression of the different PM $\mathrm{X}$ cleavage mutants.

The observation that even the quadruple mutant could efficiently cleave SUB1 and fully restore parasite growth in the absence of endogenous PM X was of particular interest. To assess the enzyme activity of the second copy PM X, we pulled down WT and Quad ${ }^{\text {mut }}$ PM X from parasites using anti-GFP antibodies followed by in vitro substrate cleavage assays. As a control, we similarly pulled down the active site 
D266G PM X mutant from parasites. The Quad ${ }^{\text {mut }}$ PM X showed robust substrate cleavage activity comparable to that of the WT at pH 5.5 (Fig. 3). For both samples the activity dropped at pH 6.5 though there was a difference in residual activity between the Quadmut and the WT enzyme. At pH 7.5, both samples lost their activity. As expected, the PM X inhibitor, CWHM-117 was able to block activity for both samples and the D266G did not show significant activity even at pH 5.5. Together, these data suggest that processing of $P M X$ is not important for its function in parasites.

\subsection{In vitro activity of rPM X is regulated by acid-induced structural changes.}

For many proteases, the $\mathrm{N}$-terminal prodomain $(\mathrm{PD})$ remains tightly bound to the catalytic domain, acting as an endogenous inhibitor for the mature enzyme ${ }^{12,13}$. Removal of the PD is therefore necessary for enzyme activation. Surprisingly we noticed that the N-terminal fragment of the proenzyme remains noncovalently attached to the processed rPM X after isolation (Fig. 1a, d). To understand this, we carried out three separate experiments. First, we analyzed WT rPM X that had been pre-incubated in different pH conditions, by native gel electrophoresis. We did not observe a low molecular weight band running separately under acidic conditions (Fig. 4a). We also performed size exclusion chromatography with WT rPM X under acidic conditions. Consistent with the native gel electrophoresis, incubation at pH 5.5 failed to separate the $\mathrm{N}$-terminal PD fragment from the rest of the protein (Fig. 4b). These data suggest that acidity-induced activation of rWT PM X does not involve complete removal of the $\mathrm{N}$ terminal part of the PD. Finally, we examined the WT rPM $X$ by circular dichroism (CD) spectroscopy. Changes in molar ellipticity specifically between the $180 \mathrm{~nm}-230 \mathrm{~nm}$ part of the spectrum suggested acidity-induced changes in the secondary structure (Fig. 4c).

\subsection{The N-terminal PD piece is required for intracellular trafficking and functionality of PMX.}

To see how the PD regulates PM X function, we made truncation mutations in the PD and expressed the truncated PM X as C-terminally GFP-tagged second copies in a SUB1-3xHA parasite line (Fig. 5a). Specifically, we either deleted the sequence between the N-terminal signal peptide (SP) and the NFLD autocleavage site (delA) or the sequence between the NFLD and the downstream IALE cleavage site (delB). As a control, we made a third construct that lacked the entire PD (delPD). IFAs with mature schizonts showed that, similar to the WT PM X, the delB but not the delA or delPD was substantially colocalized with the SUB1-3xHA in exonemes (Fig. 5b-c). We believe that partial cleavage of GFP from the PM X during secretion (Fig. 1e) accounts for the imperfect colocalization of WT and delB PM X with SUB1. The delA and deIPD, on the other hand, showed near complete colocalization with the ER marker BiP. To further assess intracellular trafficking, we looked at the secretion of second copy PM X into the culture supernatant from synchronized $41-44 \mathrm{~h}$ schizonts that were pretreated with zaprinast. Zaprinast is a phosphodiesterase (PDE) inhibitor and causes premature egress of parasites by promoting discharge of effectors from apical secretory organelles in a protein kinase $G(P K G)$-dependent manner ${ }^{14}$. Western blots from fractionated samples showed that, similar to the endogenous PM X (anti-FLAG blot), both the 
WT and delB but not delA (anti-GFP blot) were partially secreted into the culture supernatant following a 45 min zaprinast treatment (Fig. 5d). Together with the IFAs, this data therefore suggests that the Nterminal part of the PD carries information that is critical for folding and trafficking of PM $X$ beyond the ER.

In addition to trafficking, we also looked at the growth of the transgenic lines that were made in the PM $X^{\text {apt }}$ background. As shown in figure 5 e, in the absence of aTc, the growth defect observed in the parental line could be restored in the WT or delB but not the delA-expressing parasite lines. We pulled down the different second copy PM X versions from parasite lines using anti-GFP antibodies and tested their activities on fluorogenic peptide. As shown in supplementary figure 3 , the delB but not the delA PM X was active at $\mathrm{pH}$ 5.5. Therefore, the $\mathrm{N}$-terminal fragment of the PD appears to be critical for PM X activity, likely by aiding correct folding of the protease.

\subsection{Autocatalytic processing of PM X occurs in a late secretory compartment.}

To understand the maturation of PM X further, we treated synchronized 40-43 h parasites expressing PM $\mathrm{X}-3 \mathrm{xHA}$ for $8 \mathrm{~h}$ with the cysteine protease inhibitor E64d to prevent egress but still allow organellar discharge as well as PVM rupture and RBCM poration ${ }^{15,16}$. Secreted proteins (supernatants) were then separated from those that remained intercellular by centrifugation. As shown in figure 6 , similar to the vehicle (DMSO)-treated samples, both unprocessed and processed forms of PM X were detected in the culture supernatant of the E64d-treated samples. As expected, treatment with the protein kinase G (PKG) inhibitor compound 1 (C1), which blocks apical organellar discharge in P. falciparum, blocked the secretion of PMX into the culture supernatant ${ }^{17}$. Importantly, the fungal metabolite brefeldin A (BFA), which causes accumulation of newly synthesized secretory proteins in a fused ER/Golgi compartment, completely blocked PM X processing (Supplementary Fig. 4). This indicates that all the processing steps happen in a post-ER compartment.

\subsection{PM X cleaves microneme and rhoptry substrate at different cellular locations.}

The processing defect of the micronemal protein AMA1 upon knockdown of PM X (Fig. 2b) provides a genetic confirmation for the previously observed inhibition of AMA1 cleavage by a PM X-specific inhibitor ${ }^{4,5}$. The same work also identified a number of rhoptry-localized proteins as substrates of PM X. Given that PM X was originally found to be colocalized with SUB1 in the exoneme secretory organelles, these observations suggest that the interaction of PM X with its substrates may be spatially regulated. We analyzed the processing of PM X substrates under conditions where organellar discharge was blocked by treatment with $\mathrm{C} 1$. Control parasites were treated with E64d to block egress without impairing organellar discharge. Processing of AMA1 (microneme), SUB1(exoneme) and Rh5 (rhoptry) was assessed. As shown by western blot, both AMA1 and SUB1 were processed in cells treated with $\mathrm{C} 1$ (Fig. 7a). On the other hand, treatment with $\mathrm{C} 1$ blocked the processing of Rh5, resulting in the 
accumulation of the $60 \mathrm{kDa}$ precursor form (Fig. 7b). For Rh5 we could only detect the presence of the processed $50 \mathrm{kDa}$ form when parasites were allowed to discharge the apical organellar contents. As expected, all three proteins were accumulated in their precursor forms when samples were cultured in absence of aTc to knock down PM X. Finally, as a control for organellar discharge, we analyzed the processing of the PV-localized SUB1 substrate SERA5, for which processing of the $126 \mathrm{kDa}$ precursor to lower molecular weight forms was detected only when the processed SUB1 was discharged into the PV (+ aTc/+ E64d sample). Additionally, AMA1 was found to be correctly localized in terminal schizonts even in the absence of aTc, suggesting that the PM X-mediated cleavage is not necessary for trafficking to the micronemes (Supplementary Fig. 5). Altogether, the data suggest cleavage of different substrates by PM $\mathrm{X}$ is regulated spatially in $P$. falciparum.

Importantly, the fact that PM X could cleave the microneme-localized substrate AMA1 in the presence of C1 suggested that the two proteins encounter each other intracellularly. To further understand how PM X interacts with non-exoneme proteins, we carried out a series of microscopy examinations with C1-treated terminal schizonts expressing PMX-3xHA or SUB1-3xHA. In cells that are dual-labelled with anti-AMA1 and anti-HA antibodies, by epifluorescence microscopy we observed very strong colocalization between AMA1 and PM X as well as between SUB1 and AMA1 (Fig. 8a, left panel). This is of particular interest since an earlier work reported that the two proteins are targeted to different cellular compartments ${ }^{8}$. Similar results were obtained using EBA-175 as a microneme marker instead of AMA-1 (Fig. 8a, right panel). On the other hand, dual labelling of PM X-3xHA-expressing terminal schizonts with either antiRON4 (rhoptry neck marker) or with anti-RAP1 (rhoptry bulb marker) showed no overlap of signals (Fig. 8b).

Given the small size of the merozoites and the proximity of the organelles at the apical end of each merozoite, the regions of overlap observed between AMA1 and PM X or AMA1 and SUB1 could be a result of insufficient resolution by normal epifluorescence microscopy. Therefore, to better understand the localization of these proteins in schizonts, we analyzed the same dually labelled parasites by two separate high resolution imaging techniques -Airyscan (Fig. 8c and Supplementary Fig. 6a-b) and superresolution structured illumination microscopy (SR-SIM) (Supplementary Fig. 6c). Both experiments revealed differences in the punctate patterns of expression of AMA1 or EBA-175 compared to PM X or SUB1, but we also observed regions that showed complete overlap between the protein pairs. Consistent with this, when immunoelectron microscopy (immunoEM) was performed on thin-section samples, besides vesicles that were single positive for either AMA1 or PM X-3XHA, we observed vesicular structures showing signals for both proteins (Fig. 9a). Similar observations were obtained when SUB1-3xHA schizonts were used instead of PM X-3xHA schizonts (Fig. 9b). Although we could not determine whether the single-positive vesicles bud from those that contain both proteins, our data clearly indicate common partitioning of PM X and SUB1 with AMA1 inside the parasites, at least transiently.

\section{Discussion}

Autoprocessing and activity of PMX 
Aspartic proteases are canonically synthesized as proenzymes that autoactivate by self-processing at the pro-mature junction, relieving prodomain inhibition in the active site. The proenzyme form of PM X in $P$. falciparum processes itself into multiple isoforms with different $\mathrm{N}$-termini and leaves some protein unprocessed. Using both recombinant enzyme and mutant parasites we mapped the PM X internal cleavage sites. Mutations at the intra-prodomian region cleavage sites could only abolish the formation of the semi-pro form, but not the mature form and vice versa (Fig. 1e). Thus, the different truncated forms of PM X originate from independent processing events at alternative cleavage sites.

To our surprise, a quadruple mutant PM X that fails to mature, showed robust in vitro substrate cleavage activity, and could fully complement parasite growth in absence of endogenous PM X (Fig. 2-3). An ER resident protein, PfERC (Plasmodium falciparum endoplasmic reticulum-resident calcium-binding protein) has been implicated in the activation of PM X ${ }^{18}$. Knockdown of PfERC gave rise to a defect in the PM Xmediated processing step of SUB1, leading to a block in parasite egress. In those parasites, PM X accumulated in the full-length form. Our current data suggest that the observed loss of PM X functionality was likely not due to the lack of proteolytic processing.

How then is the activation of the PM X proenzyme regulated? Earlier, rPM X was shown to be active at pH $5.5^{3-5}$. However, it was not clear why acidic conditions are required for activity. Recently, two independent studies based on the crystal structures of the recombinant PfPM X reported unique folds within its PD segment ${ }^{19,20}$. Importantly, a twisted loop within the $N$ terminal part of the $P D\left(R^{95}\right.$ to $\left.G^{104}\right)$ has been shown to occupy the substrate binding pockets in the proenzyme. Additional salt bridges between the PD and the mature domain $\left(R^{95}\right.$ and $\left.E^{433}\right)$ and within the mature domain $\left(R^{244}\right.$ with the catalytic $D^{266}$ and $D^{457}$ ) have been implicated in maintaining the inactive state. Like the other autocatalytic pepsin-like proteases, therefore, a drop in $\mathrm{pH}$ has been proposed to break the salt bridges, destabilizing the inhibitory $\mathrm{PD}$, and thereby leading to PM X activation ${ }^{13}$. The $\mathrm{pH}$ dependency of $\mathrm{rPM} X$ for substrate cleavage supports this theory (Supplementary Fig. 3). Importantly, an N-terminal fragment of the PD remained noncovalently attached to the rest of the protease even under activation conditions (Fig. 4a-c). This indicates that the acidity-induced activation of PM X does not require physical separation of the PD.

Activity of parasite-derived PM X was also regulated by $\mathrm{pH}$ (Fig. 3). Nevertheless, the quadruple mutant was significantly less active than the WT PM X at pH 6.5. This suggests that while PD cleavage is not necessary, it can increase the substrate cleavage efficiency of PM X under some conditions. In parasites, however, the different cleavage mutant forms did not show a discernable defect in processing of SUB1 and AMA1 (Fig. 2b). PM X has a broad range of substrates and many of them are parasite surface adhesins ${ }^{5}$. The cleavage efficiency of the different mutant forms of PM $X$ towards these adhesins was not tested here. It is possible defects in PM X-mediated processing might alter the antigenicity of different surface adhesins. Being an intracellular pathogen that normally stays outside of its host RBC only for short period of time between egress and re-invasion, variation in antigenicity might, therefore, cost parasite replication in vivo.

Interaction of PMX with different substrates is spatially regulated. 
We showed that the PM X-mediated processing of the exoneme-localized substrate SUB1 and the microneme-localized substrate AMA1 take place exclusively inside the parasites (Fig. 7a). In contrast, the rhoptry substrate Rh5 was processed following apical organellar discharge, likely inside the PV (Fig. 7b). Given that PM X needs acidic $\mathrm{pH}$ for activity, this may indicate that the PV becomes acidic prior to egress. In the related apicomplexan parasite Toxoplasma gondii, a pH decrease in the PV towards the end of the replication cycle has been reported ${ }^{21}$.

By using two different high-resolution immunofluorescence techniques, along with immunoelectron microscopy, we observed an intimate association between the microneme and exoneme markers (Fig. 8 and Supplementary Fig. 6). Some signal was overlapping and by EM could be seen together in vesicular structures, suggesting that the two organelles might have a common base. Based on these data, therefore, we propose that the microneme and the exoneme proteins co-traffic to a precursor compartment where PM X encounters an acidic environment and subsequently cleaves its substrates. Further sorting of the proteins from such precursor compartments might result in the heterogeneity of the micronemes and exonemes.

It is still not clear why some rhoptry substrates are cleaved by PM X. In T. gondii, a PM IX/ X ortholog ASP3 has been shown to process both microneme and rhoptry substrates ${ }^{22}$. In contrast, the Plasmodium genome encodes a separate rhoptry-specific aspartic protease, plasmepsin IX (PM IX) ${ }^{3,4}$. Importantly, PM $I X$ and PM $X$ have similar substrate specificity in vitro ${ }^{5}$. This suggests that the exposure of different substrates to these proteases in Plasmodium is rather complex and might be both spatially and temporally regulated. While the molecular determinants remain to be identified, subcompartmentalization of different proteins inside the rhoptries have been documented ${ }^{23,24}$. It is believed that this organization regulates interaction between different proteins within the rhoptries and as well as the timing of protein secretion. Rh5 along with other Rhs are located at the very tip of the rhoptries where PM IX is excluded, and therefore, it is possible that Rh5 is not accessible to PM IX ${ }^{3,4,25}$. Moreover, while it remains unknown how PM X-mediated cleavage regulates Rh5 function, we have shown that PM X is secreted inside the PV (Fig. 6), where we propose that the cleavage happens. Whether PM IX is a secreted protease remains unknown.

\section{Materials And Methods}

\subsection{Reagents}

All primers were obtained from Integrated DNA Technologies. Restriction enzymes were

purchased from New England Biolabs. Compound 1 was purchased from MedChem Express. E64d and zaprinast were purchased from Sigma. Ni-NTA beads, anti-HA antibody-coated magnetic beads and the mouse-anti HA antibody were from ThermoFisher; the anti-GFP coated magnetic beads were from Chromotek. The rat anti-FLAG antibody was from Novus Biologicals, the rabbit anti-HA antibody and the rabbit anti-His antibody were from Sigma, the rabbit anti-AMA1 antibody was from LSBio, the mouse anti- 
GFP antibody was from Invitrogen. Monoclonal antibodies 2.29 (anti-RAP-1) and 12.4 (anti MSP-1) were obtained from The European Malaria Reagent Repository (http://www.malariaresearch.eu). The anti-EBA175 antibody (MRA-2) and the anti-BiP antibody were from MR-4. The mouse anti-PM $V$ antibody was previously described ${ }^{26}$. Anhydrotetracycline (aTc) was purchased from Cayman Chemical. Synthetic PM $X$ substrate peptides were from Eurogentec.

\subsection{Generation of plasmids}

To complement the PM X knockdown, we modified the previously described pyEOE-2X attP-3X MYC vector $^{3}$. At first, the yDHOD cassette was replaced with the human dihydrofolate reductase (hDHFR), resulting into the vector $\mathrm{pEOE}-2 \mathrm{X}$ attP. To make PM X expression stage-specific, we used a $1700 \mathrm{bp}$ sequence located upstream of the AMA1 coding region. This promoter (pAMA1) has been successfully used for transgenic expression of other late-stage specific genes in $P$. falciparum ${ }^{10,27}$. The pAMA1 was amplified from genomic DNA of the NF54 strain of $P$. falciparum using the primer pair 151 and 152 and subsequently gel extracted. The purified fragment was ligated between the Aflll and Xhol restriction enzymes cut sites within pEOE-2X attP generating pEOE-pAMA1-2X attP. The ORF of PM $X$ was amplified using the primers 153 and 154. The ORF of green fluorescence protein (GFP) was PCR-amplified from the plasmid PM2GT ${ }^{28}$ using the primers 155 and 156. Amplified PM X and GFP ORFs were subsequently fused by Gibson assembly with the pEOE-pAMA1-2X attP that was previously digested with Xhol and Eagl. This resulted in the production of the final WT complementation construct pEOE-PM X-GFP-2XattP. Subsequent site-directed mutagenesis (QuickChange lightning, Agilent) on this plasmid used primers 157, 158,159 and 160 to generate the different PM X cleavage mutant constructs. For generating the different PD truncation mutant constructs, pEOE-PM X-GFP plasmid was subjected to site-directed mutagenesis.

Mutagenesis primers 161 and 162 were used to generate the delA and delB constructs respectively. DelPD construct was made in a similar way using primer 163. Tagging the endogenous ORF of SUB1 with 3xHA was carried out using previously published plasmids ${ }^{3}$. The different cleavage mutant constructs for recombinant PM X were made from the parental construct using the primers shown in table S1.

\subsection{Parasite culture and transfection and synchronization}

NF54attB parasites ${ }^{29}$ and resultant transgenic strains were cultured in human red blood cells as previously described ${ }^{30}$. PM X ${ }^{\text {apt }}$ parasites ${ }^{3}$ were maintained in presence of $100 \mathrm{nM}$ aTc and $2.5 \mu \mathrm{g} / \mathrm{ml}$ BSD. For complementation of PM X, plasmids carrying WT or mutant PMX coding sequences were independently co-transfected with a Bxb1 integrase plasmid ${ }^{31}$ into the $\mathrm{PMX}^{\text {apt }}$ parasites. Parasites were selected with $5 \mathrm{nM}$ WR, together with $2.5 \mu \mathrm{g} / \mathrm{ml} \mathrm{BSD}$ and $1 \mu \mathrm{M}$ aTc. The integration of these plasmids into the PMX ${ }^{\text {apt }}$ strain was confirmed by PCR using the primer pair 164 and 165 . For tagging the 3 end of SUB1, the NF54attB parasites were cotransfected with the yPM2GT-SUB1-3xHA and the PAIO3 guide plasmids. The transfectants were selected in the presence of $12.5 \mu \mathrm{g} / \mathrm{ml}$ DSM1. Integration of the plasmid was verified by PCR.

Highly synchronous ring-stage parasites were obtained as follows. High-parasitemia schizont cultures were passed through MACS LD magnet columns (Miltenyi Biotec) and schizonts were collected. These 
were then added to fresh uninfected RBCs resuspended in warm culture media. The cultures were shaken at 80 RPM for $3 \mathrm{~h}$ and the resulting parasites were synchronized using $5 \%$ sorbitol as described before ${ }^{32}$.

\subsection{Parasite growth assay}

For growth curves, synchronized ring-stage parasites were washed extensively to remove residual aTc and were diluted to an initial $1 \%$ parasitemia at $2 \%$ hematocrit. Cultures were split and aTc was added to appropriate wells. Parasitemia was determined every $24 \mathrm{~h}$ using flow cytometry as described previously ${ }^{3}$.

\subsection{Natural and zaprinast-induced parasite egress assays}

To follow natural parasite egress, 41-44 h schizonts expressing PM X-3xHA were MACS- purified and treated with either vehicle (DMSO) or with $\mathrm{E} 64 \mathrm{~d}(10 \mu \mathrm{M})$ for $8 \mathrm{~h}$. A third sample was treated similarly with the PKG inhibitor $\mathrm{C} 1(1.5 \mu \mathrm{M})$ to block discharge from the apical organelles ${ }^{14}$. Following incubation, the discharged proteins (in the culture supernatant) were separated from those that remained intracellular by centrifuging the cultures at $10,000 \mathrm{~g}$ for $10 \mathrm{~min}$. PM X was subsequently pulled down using anti-HA beads and subjected to western blot.

Zaprinast-induced organellar discharge was performed as described previously ${ }^{14}$. Briefly, $41-44 \mathrm{~h}$ synchronized parasites were MACS purified and transferred to albumax-free medium. Zaprinast $(75 \mu \mathrm{M})$ was added to the cultures and samples were either harvested immediately $(0 \mathrm{~min})$ or after $45 \mathrm{~min}$ of incubation. Culture supernatants were separated from the intracellular fractions as mentioned.

Endogenous or second copy PM $X$ was pulled down from both fractions using anti-FLAG and anti-GFP antibodies respectively, followed by western blot.

\subsection{Brefeldin A sensitivity assay}

To determine the effect of brefeldin A (BFA) on the processing of PM X, a pulse-chase assay was performed as before ${ }^{33}$. Briefly, synchronized cultures of schizont-infected RBCs were washed once in RPMI without methionine and cysteine, resuspended in labeling medium (RPMI without methionine and cysteine) containing $250 \mu \mathrm{Ci} / \mathrm{ml}$ [35S]methionine-cysteine (PerkinElmer) and incubated at $37^{\circ} \mathrm{C}$ for $5 \mathrm{~min}$. Cultures were either harvested immediately or maintained in label-free complete medium containing 10 $\mu \mathrm{g} / \mathrm{ml}$ cycloheximide for $60 \mathrm{~min}$ at $37^{\circ} \mathrm{C}$ before harvest. During incubation, BFA ( $5 \mu \mathrm{g} / \mathrm{ml}$ ) was added to one culture. Subsequently, PM X-3xHA was pulled down, resolved by SDS-PAGE and subjected to autoradiography.

\subsection{Expression and purification of recombinant PM X (rPM $X$ ) from mammalian cells}

The rPM X expression vector for mammalian cells was a gift from UCB Pharma, USA. In this construct, a mammalian recodonized version of PM X was $\mathrm{C}$-terminally tagged with $8 \mathrm{x}$-histidine (8x-His) and the endogenous signal peptide at the $\mathrm{N}$-terminal end was replaced with a mammalian signal peptide. For protein expression, the Freestyle 293-F cells (Thermo Fisher) were transfected with a mixture of DNA and polyethylenimine (1:3). The rPM X was expressed as a secreted protein. $72 \mathrm{~h}$ post transfection the 
supernatant was harvested. The diluted supernatant (1: 3 in $50 \mathrm{mM}$ Tris-Cl pH 8.0, $100 \mathrm{mM} \mathrm{NaCl}, 10 \mathrm{mM}$ imidazole) was purified using Ni-NTA resin (ThermoFisher). After extensive wash in the same buffer, the protein was eluted with $50 \mathrm{mM}$ Tris- $\mathrm{Cl} \mathrm{pH} \mathrm{8.0,100} \mathrm{mM} \mathrm{NaCl,} 400 \mathrm{mM}$ imidazole. The eluted sample was then buffer exchanged using an Amicon ultra $3 \mathrm{kDa}$ molecular wt. cutoff filter (Millipore, USA) and finally resuspended in $50 \mathrm{mM}$ Tris $\mathrm{pH} 7.5,100 \mathrm{mM} \mathrm{NaCl}$. If not used immediately, $1 \%$ glycerol was added and the preparation was stored in $-80^{\circ} \mathrm{C}$.

\subsection{Circular dichroism (CD) spectroscopy assay}

To examine the secondary structure of rPM X, samples were diluted to $35 \mathrm{ug} / \mathrm{ml}$ in $10 \mathrm{mM}$ potassium phosphate buffer with indicated pHs. CD spectra were recorded on a JASCO-J715 polarimeter (JASCO, Tokyo, Japan) over the wavelength range $185-325 \mathrm{~nm}$ in a 1-mm path length quartz cuvette using a step size of $0.5 \mathrm{~nm}$, a slit bandwidth of $1.0 \mathrm{~nm}$, and a signal averaging time of $1.0 \mathrm{~s}$. For each wavelength three scans were performed. AVIV software was used for background subtraction. Molar ellipticity [ $\theta$ ] was calculated using the formula: $[\theta]=\theta(\mathrm{mdeg}) / 10 \bullet \mathrm{C}(\mathrm{mol} / \mathrm{lit}) \bullet(\mathrm{cm})$, where $\mathrm{C}$ is the concentration of the sample, $\mathrm{I}$ is the path length.

\subsection{In vitro substrate cleavage assay}

The ability of rPM X to cleave fluorogenic substrate peptides was performed as described ${ }^{4,5}$. Briefly, $20 \mathrm{ng}$ of $r P M X$ was incubated with $1 \mu \mathrm{M}$ DABCYL-HSFIQEGKE-E-EDANS (Rh2N peptide) in $200 \mu \mathrm{l}$ of PM X activity buffer $(25 \mathrm{mM}$ MES, $25 \mathrm{mM}$ Tris, $\mathrm{pH} 5.5)$ for $90 \mathrm{~min}$ at $37^{\circ} \mathrm{C}$. As a negative control, a point mutant form of the peptide that abolishes PM X-mediated cleavage (DABCYL-HSFAAEGKE-E-EDANS) was used as a substrate. To inhibit PM X activity, $1 \mu \mathrm{M}$ CWHM-117 3,34 was added to samples at pH 5.5. Each sample was analyzed in triplicate using a Synergy HTX microplate reader at $340 \mathrm{~nm}$ wavelength. Readings were taken at 1-min intervals. PM X activity was measured as an increase in the relative fluorescence units (RFUs) over time.

To determine the activity of parasite-derived PM X, synchronized $42-45 \mathrm{~h}$ schizonts $\left(2 \times 10^{7}\right.$ cells per sample) expressing PM X-GFP were lysed in ice-cold native lysis buffer (50 mM Tris, pH 7.5, $150 \mathrm{mM}$ $\mathrm{NaCl}, 1 \% \mathrm{NP}-40$ ). PM X-GFP was pulled down from whole cell lysates using anti-GFP antibody-coated magnetic beads (Chromotek) according to the manufacturer's recommendations. Following washes (4x) in the lysis buffer, beads were incubated with the Rh2N substrate peptide $(1 \mu \mathrm{M})$ in $200 \mu \mathrm{L}$ of PM X activity buffer. Fluorescence readings were taken either immediately or following $1 \mathrm{~h}$ incubation in $37^{\circ} \mathrm{C}$.

\subsection{Liquid chromatography/ Mass spectrometry (LC/MS)}

To determine the cleavage sites of the processed products in $\mathrm{PPM} \mathrm{X}$, the indicated bands in figure $1 \mathrm{~A}$ were excised from the gel and were subjected to LC/MS analysis. Briefly, the gel samples were destained, reduced and alkylated prior to trypsin digestion. Digest was aspirated and combined with further extraction of the gel piece with $60 \%$ acetonitrile (ACN) in $1 \%$ trifluoroacetic acid (TFA). The extracted peptides were dried down and each sample was resuspended in $10 \mu \mathrm{L} 5 \mathrm{ACN} / 0.1 \%$ formic acid (FA). 5 $\mu \mathrm{L}$ was analyzed by LC-MS with a Dionex RSLCnano HPLC coupled to an Orbitrap Fusion Lumos (Thermo 
Scientific) mass spectrometer using a $2 \mathrm{~h}$ gradient. Peptides were resolved in a $75 \mu \mathrm{m}$ x $50 \mathrm{~cm}$ PepMap C18 column (Thermo Scientific) with following gradient: Time $=0-4 \mathrm{~min}, 2 \% \mathrm{~B}$ isocratic; $4-8 \mathrm{~min}, 2-10 \%$ B; 8-83 min, 10-25\% B; 83-97 min, 25-50\% B; 97-105 min, 50-98\%. Mobile phase consisted of A, 0.1\% FA; mobile phase B, $0.1 \%$ FA in ACN. The instrument was operated in the data-dependent acquisition mode in which each MS1 scan was followed by Higher-energy collisional dissociation (HCD) of as many precursor ions in 2 second cycle (Top Speed method). The mass range for the MS1 done using the FTMS was 365 to $1800 \mathrm{~m} / \mathrm{z}$ with resolving power set to 60,000 at $400 \mathrm{~m} / \mathrm{z}$ and the automatic gain control (AGC) target set to $1,000,000$ ions with a maximum fill time of $100 \mathrm{~ms}$. The selected precursors were fragmented in the ion trap using an isolation window of $1.5 \mathrm{~m} / \mathrm{z}$, an AGC target value of 10,000 ions, a maximum fill time of $100 \mathrm{~ms}$, a normalized collision energy of 35 and activation time of $30 \mathrm{~ms}$. Dynamic exclusion was performed with a repeat count of 1 , exclusion duration of $30 \mathrm{~s}$, and a minimum MS ion count for triggering MS/MS set to 5000 counts.

\section{Data Analysis}

All MS/MS samples were analyzed using Mascot (Matrix Science, London, UK; version 2.5.1.0). Mascot was set up to search against provided sequence along with common contaminants. The digestion enzyme was set as semiTrypsin (to account for nontryptic termini). Mascot was searched with a fragment ion mass tolerance of $0.60 \mathrm{Da}$ and a parent ion tolerance of $10 \mathrm{ppm}$. Oxidation of methionine, carbamidomethylation of cysteine, and acetylation of N-terminal of protein were specified in Mascot as variable modifications. Scaffold (version Scaffold_4.8.2 Proteome Software Inc., Portland, OR) was used to validate MS/MS based peptide and protein identifications. Peptide identifications were accepted if they could be established under $1 \%$ FDR by the Peptide Prophet algorithm ${ }^{35}$ with Scaffold delta-mass correction. Protein identifications were accepted if they could be established at greater than $99.0 \%$ probability and contained at least 2 identified peptides. Protein probabilities were assigned by the Protein Prophet algorithm ${ }^{36}$.

\subsection{Size exclusion chromatography}

For gel filtration, rPM X samples were pretreated in acidic PM X activation buffer ( $25 \mathrm{mM}$ MES, $25 \mathrm{mM}$ Tris, pH 5.5) for $30 \mathrm{~min}$ at room temperature. Before sample runs, the Superdex 200 10/300 column (GE Healthcare) was equilibrated in the same buffer. Following runs, the samples were collected as $0.5 \mathrm{ml}$ fractions. Fractions of interest (as indicated in Fig. 4) were further concentrated using an Amicon ultra 3 kDa mol. wt. cutoff filter. Finally, samples were resolved by SDS-PAGE and the gel was stained with Coomassie dye. For gel filtration under denaturation, every step was done as mentioned except that the buffers were supplemented with $6 \mathrm{M}$ guanidine hydrochloride. For denaturation, sample was treated with $6 \mathrm{M}$ guanidine hydrochloride and heated at $60^{\circ} \mathrm{C}$ for $45 \mathrm{~min}$ before loading onto the size exclusion column.

4.12 Native gel electrophoresis

Page $13 / 26$ 
For native PAGE, rPM X was preincubated in buffers ( $25 \mathrm{mM}$ MES and $25 \mathrm{mM}$ Tris) with indicated $\mathrm{pH}$ conditions for $1 \mathrm{~h}$ at RT. Samples were then mixed with $5 \mathrm{x}$ native gel loading buffer ( $312.5 \mathrm{mM}$ Tris, $\mathrm{pH}$ $6.8,50 \%$ glycerol, $0.05 \%$ bromophenol blue) and run in a discontinuous gel with $4 \%$ and $10 \%$ polyacrylamide for the stacking and the resolving parts respectively. Sodium dodecyl sulphate (SDS) was excluded both from the gel and the running buffer (192 mM glycine, $25 \mathrm{mM}$ Tris, pH 8.3). Gel was subsequently stained with Coomassie brilliant blue R250 (ThermoFisher) and destained in $30 \%$ methanol, $10 \%$ glacial acetic acid.

\subsection{Western blots}

For PM X knockdown western blots (Fig. 2b), following synchronization, each culture was split into two plates. To one plate, aTc was added, and to the other equal volume of vehicle (DMSO) was added. $46 \mathrm{~h}$ post invasion, schizonts were collected by saponin lysis and stored at $-80^{\circ} \mathrm{C}$ until further processing. Samples were subsequently lysed in RIPA buffer supplemented with protease inhibitor cocktail and hemozoin was removed by centrifugation. Cell lysates were mixed with $4 x$ sample loading buffer and heated at $99^{\circ} \mathrm{C}$ for $5 \mathrm{~min}$. Fractions of equal cell equivalents were subjected to SDS-PAGE and immunoblotting.

For processing inhibition assays (Fig. 7), PM X $\mathrm{X}^{\text {apt }}$ parasites were synchronized and grown up to $44 \mathrm{~h}$ of age, as above. The infected RBCs from + aTc were then separated by MACS and resuspended in a small volume supplemented with either E64d $(10 \mu \mathrm{M})$ or with compound $1(1.5 \mu \mathrm{M})$ for the next $6 \mathrm{~h}$. Cultures were then separated from the culture supernatant and the pellet fractions were boiled with $2 x$ sample loading buffer.

Primary antibodies included rabbit anti-AMA1 (1:500), rabbit anti-SUB1 (1:1000), rabbit anti-HA (1:1000), rabbit anti-Sera5 (1:1000), mouse anti PM V (1:500), rabbit anti-Rh5 (1:500), mouse anti-GFP (1:1000), mouse anti-his (1:1000), and rat anti-FLAG (1:1000). For all, appropriate IRDye conjugated secondary antibodies were used at 1:10000 dilution. Blots were visualized on an Odyssey imaging system (Licor).

\subsection{Immunofluorescence assays}

For IFAs, cells were fixed as described previously ${ }^{37}$. Briefly, synchronized and C1-treated mature schizonts were fixed in 3.7\% paraformaldehyde for 15 min and blocked in $3 \% \mathrm{BSA}$ in PBS overnight at $4^{\circ} \mathrm{C}$ before antibody staining. The antibodies used for IFA were: rabbit anti-HA (Invitrogen, 1:500), mouse anti-HA (1:500), mouse anti-GFP (Invitrogen;1:500), mouse anti-MSP1 (1:500), rabbit anti-AMA1 (1:500), mouse anti-RAP1 (1:500), rabbit anti-EBA175 (1:500), rabbit anti-RON4 (1:500) and rabbit anti-BiP (1:250). The secondary antibodies were used as 1:2000 dilutions and were conjugated to Alexa Fluor 488 or 546 (Life Technologies). Cells were mounted with ProLong and 4',6'-diamidino-2-phenylindole (DAPI) (Invitrogen) and imaged using a Zeiss Imager M2 Plus wide field fluorescence microscope, using a 63x objective for epifluorescence imaging. 
For confocal imaging, samples were analyzed with a Zeiss LSM880 laser scanning confocal microscope with Airyscan (Carl Zeiss Inc. Thornwood, NY). A Plan-Apochromat 63X (NA 1.4) DIC objective and ZEN black software (version 2.1 SP3) were used for image acquisition. The image analysis software Volocity (version 6.3) (PerkinElmer, Waltham, MA) was used for 3-dimensional rendering of $Z$ slices acquired through the depth of the parasites. For SR-SIM microscopy, samples were analyzed with a Nikon SIM super resolution microscope using a 100x objective. Image processing, analysis, and display were performed using Axiovision or Zen Blue. Adjustments to brightness and contrast were made for display purposes.

\subsection{Immunoelectron microscopy}

Samples for immunoEM were prepared as before ${ }^{3}$. Briefly, the infected RBCs were fixed in $4 \%$ paraformaldehyde (Polysciences Inc., Warrington, PA) with $100 \mathrm{mM} \mathrm{PIPES} / 0.5 \mathrm{mM} \mathrm{MgCl}_{2}, \mathrm{pH} 7.2$ for $1 \mathrm{~h}$ at $4^{\circ} \mathrm{C}$. Samples were then embedded in $10 \%$ gelatin and infiltrated overnight with $2.3 \mathrm{M}$ sucrose $/ 20 \%$ polyvinyl pyrrolidone in PIPES/MgCl2 at $4^{\circ} \mathrm{C}$. Samples were trimmed, frozen in liquid nitrogen, and sectioned with a Leica Ultracut UCT cryo ultramicrotome (Leica Microsystems Inc., Bannockburn, IL). 50 nm sections were blocked with $5 \% \mathrm{FBS} / 5 \%$ NGS for 30 min and subsequently incubated with primary antibodies for $1 \mathrm{~h}$, followed by secondary antibodies conjugated to $12 \mathrm{~nm}$ or $18 \mathrm{~nm}$ colloidal gold (1:30) for $1 \mathrm{~h}$. Sections were washed in PIPES buffer followed by a water rinse, and stained with $0.3 \%$ uranyl acetate/2\% methyl cellulose and viewed on a JEOL 1200EX transmission electron microscope (JEOL USA, Peabody, MA) equipped with an AMT 8 megapixel digital camera (Advanced Microscopy Techniques, Woburn, MA). All labeling experiments were conducted in parallel with controls omitting the primary antibody, which were consistently negative at the concentration of colloidal gold-conjugated secondary antibodies used in these studies.

\subsection{Statistical analysis}

Unless specified otherwise, assay values in all figures are averaged from three independent repeats and error bars represent standard deviations. Differences were assessed by the Student's t-test using the Microsoft Excel software. For quantification of colocalization in figure $5 c$, epifluorescence images were analyzed by ImageJ and a Pearson's colocalization coefficient was determined for the indicated antigen pairs. $P$ values indicating statistical significance were grouped in all figures $(\star \star \star: p<0.001, \star *: p<0.01, *$ : $p<0.05)$.

\section{Declarations}

\section{Author contributions}

S.M. and D.E.G conceived and designed the study. S.M. performed research, acquired, and analyzed data. E.S. acquired data. S.M. and D.E.G wrote the manuscript. 


\section{Acknowledgments}

This work was supported by grant Al138447 from the National Institute of Allergy and Infectious Diseases (NIAID) to D.E.G. We thank Dr. Wandy Beatty (WUSTL) for immune-electron microscopy, the Molecular Microbiology Imaging Facility at WUSTL for confocal Airyscan microscopy and the Washington University Center for Cellular Imaging (WUCCI) for SR-SIM microscopy, the Proteomics \& Mass Spectrometry Facility at the Danforth Plant Science Center for LC/MS data acquisition and analysis, Dr. Bob Krantz (WUSTL) and Dr. David Sibley (WUSTL) for usage of the JASCO-J715 polarimeter and the Synergy HTX microplate reader respectively, Dr. Michael J. Blackman (The Francis Crick Institute, London) for anti-SUB1 and SERA5 antibodies, Dr. Jean-François Dubremetz (University of Montpellier, France) for anti-RON4 antibody and Dr. Eizo Takashima (Ehime University, Japan) for antiRh5 antibody. For $\mathrm{rPM} X$ production, the codon-engineered PM X gene and protocols were provided by Don Lorimer (UCB). We also thank Barb Vaupel for assistance with cloning, Dr. Darya Urusova (WUSTL) for gel filtration assays, Dr. Eva Istvan, Dr. Sebastian Nasamu and Dr. Alex Polino for useful suggestions.

\section{References}

1. WHO. WHO World Malaria Report 2021.

2. Autino, B., Corbett, Y., Castelli, F. \& Taramelli, D. Pathogenesis of malaria tissues and blood. Mediterr. J. Hematol. Infect. Dis. 4, e2012061 (2012).

3. Nasamu, A. S. et al. Plasmepsins IX and X are essential and druggable mediators of malaria parasite egress and invasion. Science 358, 518-522 (2017).

4. Pino, P. et al. A multistage antimalarial targets the plasmepsins $I X$ and $X$ essential for invasion and egress. Science 358, 522-528 (2017).

5. Favuzza, P. et al. Dual Plasmepsin-Targeting Antimalarial Agents Disrupt Multiple Stages of the Malaria Parasite Life Cycle. Cell Host Microbe 27, 1-17 (2020).

6. Thomas, J. A. et al. A protease cascade regulates release of the human malaria parasite Plasmodium falciparum from host red blood cells. Nat. Microbiol. 3, 447-455 (2018).

7. Das, S. et al. Processing of Plasmodium falciparum Merozoite Surface Protein MSP1 Activates a Spectrin-Binding Function Enabling Parasite Egress from RBCs. Cell Host Microbe 28, 433-444 (2015).

8. Yeoh, S. et al. Subcellular Discharge of a Serine Protease Mediates Release of Invasive Malaria Parasites from Host Erythrocytes. Cell 131, 1072-1083(2007).

9. Healer, J., Crawford, S., Ralph, S., McFadden, G. \& Cowman, A. F. Independent translocation of two micronemal proteins in developing Plasmodium falciparum merozoites. Infect. Immun. 70, 57515758 (2002).

10. Child, M. A. et al. Molecular determinants for subcellular trafficking of the malarial sheddase PfSUB2. Traffic 14, 1053-1064 (2013). 
11. Counihan, N. A., Kalanon, M., Coppel, R. L. \& De Koning-Ward, T. F. Plasmodium rhoptry proteins: Why order is important. Trends Parasitol. 29, 228-236 (2013).

12. Pandey, K. C., Sijwali, P. S., Singh, A., Na, B.-K. \& Rosenthal, P. J. Independent intramolecular mediators of folding, activity, and inhibition for the Plasmodium falciparum cysteine protease falcipain-2. J. Biol. Chem. 279, 3484-3491 (2004).

13. Khan, A. R., Khazanovich-Bernstein, N., Bergmann, E. M. \& James, M. N. G. Structural aspects of activation pathways of aspartic protease zymogens and viral $3 \mathrm{C}$ protease precursors. Proc. Natl. Acad. Sci. U. S. A. 96, 10968-10975 (1999).

14. Collins, C. R. et al. Malaria Parasite cGMP-dependent Protein Kinase Regulates Blood Stage Merozoite Secretory Organelle Discharge and Egress. PLoS Pathog. 9, e1003344 (2013).

15. Glushakova, S., Mazar, J., Hohmann-marriott, M. F. \& Hama, E. Malaria Parasites From Infected Erythrocytes. Cell 11, 95-105 (2010).

16. Wickham, M. E., Culvenor, J. G. \& Cowman, A. F. Selective inhibition of a two-step egress of malaria parasites from the host erythrocyte. J. Biol. Chem. 278, 37658-37663 (2003).

17. Taylor, H. M. et al. The malaria parasite cyclic GMP-dependent protein kinase plays a central role in blood-stage schizogony. Eukaryot. Cell 9, 37-45 (2010).

18. Fierro, M. A. et al. An Endoplasmic Reticulum CREC Family Protein Regulates the egress proteolytic cascade in malaria parasites. mBio 11, e03078-19 (2020).

19. Hodder, A. N., Scally, S., Triglia, T., Dietrich, M. H. \& Olsen, D. Basis for drug selectivity of plasmepsin IX and X inhibition for Plasmodium falciparum and vivax. http://dx.doi.org/10.2139/ssrn.3948205 (2021).

20. Kesari, P. et al. Structures of plasmepsin X from P. falciparum reveal a novel inactivation mechanism of the zymogen and molecular basis for binding of inhibitors in mature enzyme. bioRxiv 2021.09.24.460453 (2021).

21. Roiko, M. S., Svezhova, N. \& Carruthers, V. B. Acidification Activates Toxoplasma gondii Motility and Egress by Enhancing Protein Secretion and Cytolytic Activity. PLoS Pathog. 10, e1004488 (2014).

22. Dogga, S. K. et al. A druggable secretory protein maturase of Toxoplasma essential for invasion and egress. Elife 6, e27480 (2017).

23. Richard, D. et al. Identification of rhoptry trafficking determinants and evidence for a novel sorting mechanism in the malaria parasite Plasmodium falciparum. PLoS Pathog. 5, e1000328 (2009).

24. Baum, J. et al. Reticulocyte-binding protein homologue 5 - An essential adhesin involved in invasion of human erythrocytes by Plasmodium falciparum. Int. J. Parasitol. 39, 371-380 (2009).

25. Counihan, N. A., Kalanon, M., Coppel, R. L. \& de Koning-Ward, T. F. Plasmodium rhoptry proteins: why order is important. Trends Parasitol. 29, 228-236 (2013).

26. Banerjee, R. et al. Four plasmepsins are active in the Plasmodium falciparum food vacuole, including a protease with an active-site histidine. Proc. Natl. Acad. Sci. U. S. A. 99, 990-995 (2002). 
27. Olivieri, A. et al. Juxtamembrane shedding of Plasmodium falciparum AMA1 is sequence independent and essential, and helps evade invasion-inhibitory antibodies. PLOS Pathog. 7, e1002448 (2011).

28. Klemba, M., Beatty, W., Gluzman, I. \& Goldberg, D. E. Trafficking of plasmepsin II to the food vacuole of the malaria parasite Plasmodium falciparum. J. Cell Biol. 164, 47-56 (2004).

29. Adjalley, S. H. et al. Quantitative assessment of Plasmodium falciparum sexual development reveals potent transmission-blocking activity by methylene blue. Proc. Natl. Acad. Sci. U. S. A. 108, e1214-23 (2011).

30. Istvan, E. S. et al. Esterase mutation is a mechanism of resistance to antimalarial compounds. Nat. Commun. 8, 14240 (2017).

31. Nkrumah, L. J. et al. Efficient site-specific integration in Plasmodium falciparum chromosomes mediated by mycobacteriophage Bxb1 integrase. Nat. Methods 3, 615-621 (2006).

32. Lambros, C. \& Vanderberg, J. P. Synchronization of Plasmodium falciparum erythrocytic stages in culture. J. Parasitol. 65, 418-420 (1979).

33. Banerjee, R., Francis, S. E. \& Goldberg, D. E. Food vacuole plasmepsins are processed at a conserved site by an acidic convertase activity in Plasmodium falciparum. Mol. Biochem. Parasitol. 129, 157165 (2003).

34. Meyers, M. J. et al. Evaluation of aminohydantoins as a novel class of antimalarial agents. ACS Med. Chem. Lett. 5, 89-93 (2014).

35. Keller, A., Nesvizhskii, A. I., Kolker, E. \& Aebersold, R. Empirical statistical model to estimate the accuracy of peptide identifications made by MS/MS and database search. Anal. Chem. 74, 53835392 (2002).

36. Nesvizhskii, A. I., Keller, A., Kolker, E. \& Aebersold, R. A statistical model for identifying proteins by tandem mass spectrometry. Anal. Chem. 75, 4646-4658 (2003).

37. Absalon, S. et al. Calcium-Dependent Protein Kinase 5 Is Required for Release of Egress-Specific Organelles in Plasmodium falciparum. mBio 9, e00130-18 (2018).

\section{Figures}


a

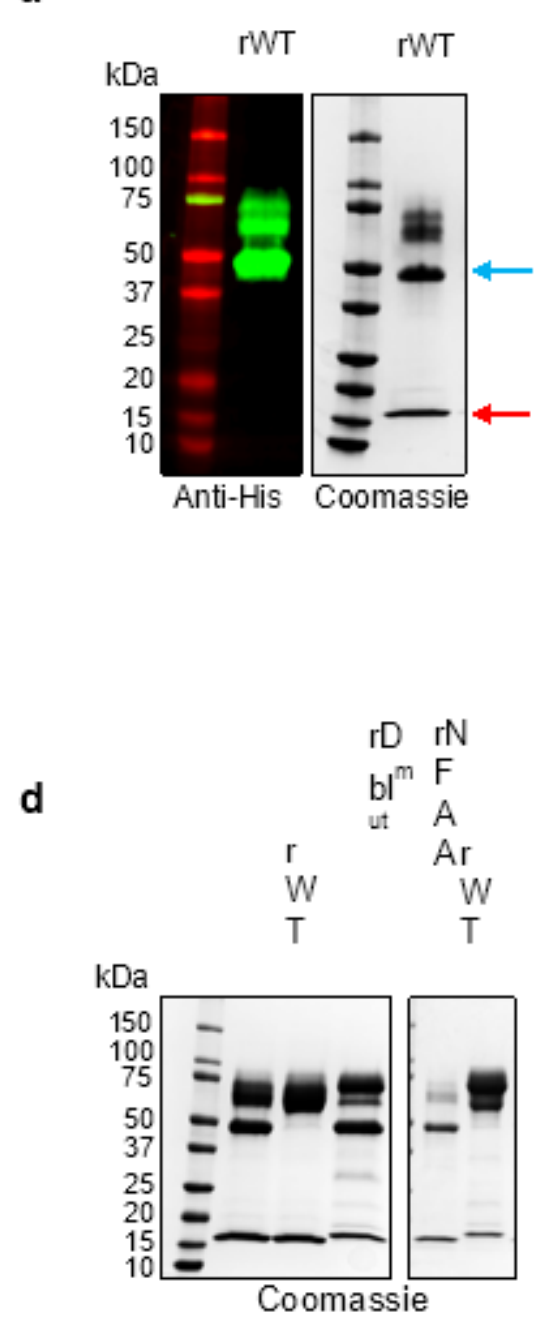

b

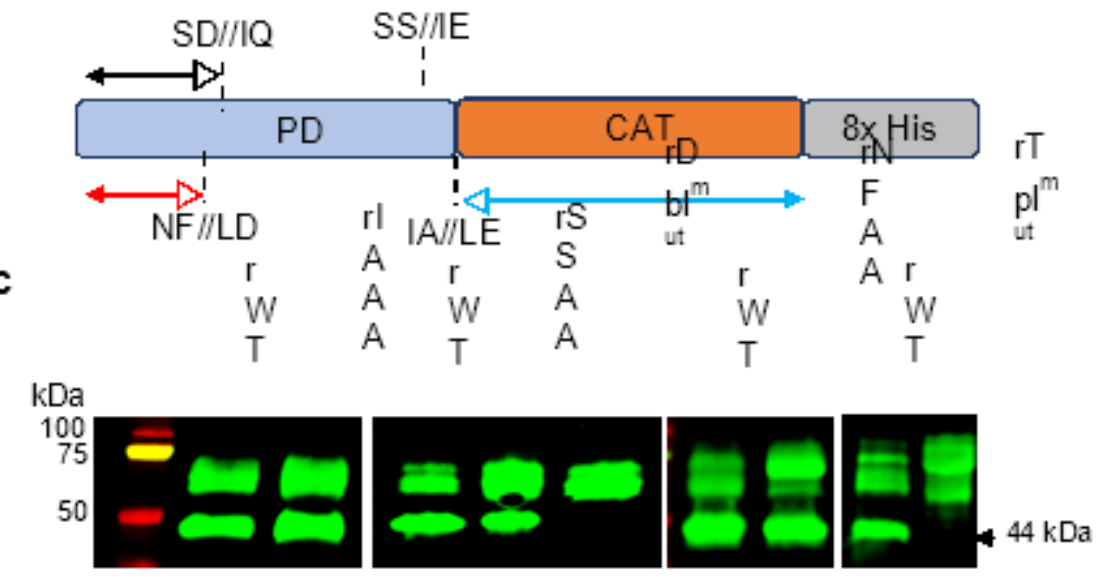

IB: anti-His

W NF SS Qu

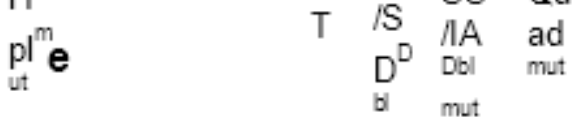

mut

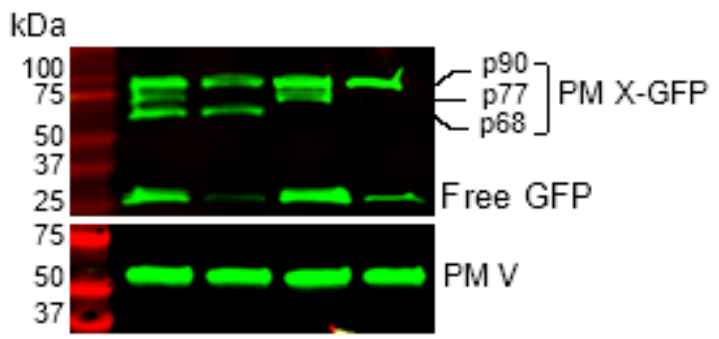

Fig. 1

\section{Figure 1}

Alternative autoprocessing sites are conserved between the heterologously expressed and parasite PM X. a PM X was purified as a C-terminally 8x His tagged secreted protein from the mammalian HEK293 cells. Left, anti-His tag immunoblot; right, Coomassie stain. In the Coomassie gel a $16 \mathrm{kDa}$ N-terminal peptide (red arrow) was detected. Blue arrow: $44 \mathrm{kDa}$ mature form of rPM X. b Schematic of the rPM X with the autocatalytic tetrapeptides indicated. Arrow headed lines represent the peptide coverage regions obtained from LC/MS. Filled arrow heads: tryptic ends, empty arrow heads: non-tryptic ends as identified by LC/MS. Red and blue arrows correspond to the bands highlighted by same color arrows in a. $\mathbf{c}$ and $\mathbf{d}$ Western blot with anti-his antibody (c) and Coomassie gel (d) showing processing of wild-type (WT) or mutant $\mathrm{rPM} X$ at multiple alternative autocleavage sites. rDbI $^{\text {mut. }}$ IAAA/SSAA double mutant; $r$ TpI ${ }^{\text {mut. }}$. double mutant with additional NFAA mutation. e Expression of second-copy PM X in parasites. Top panel: blotted with anti-GFP antibody, the molecular weights of both unprocessed and differentially processed 
forms indicated. Bottom panel: lysates from same samples but blotted with anti-PM V antibody as a loading control. Quadmut : quadruple mutant with all four cleavage sites changed to AA in the P1' and P2' positions. Each experiment was repeated at least three times and shown are representative blots.

a

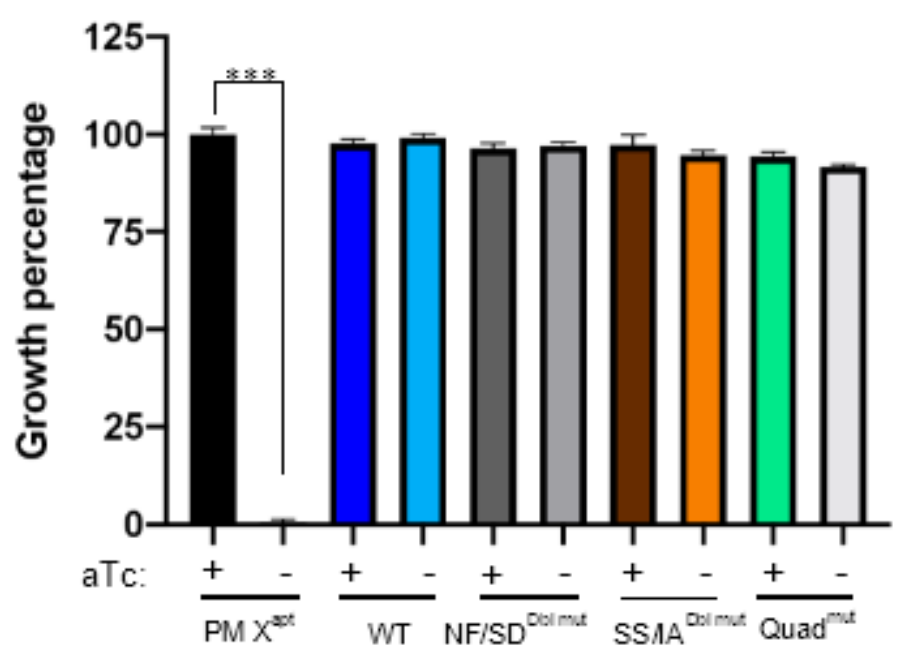

b

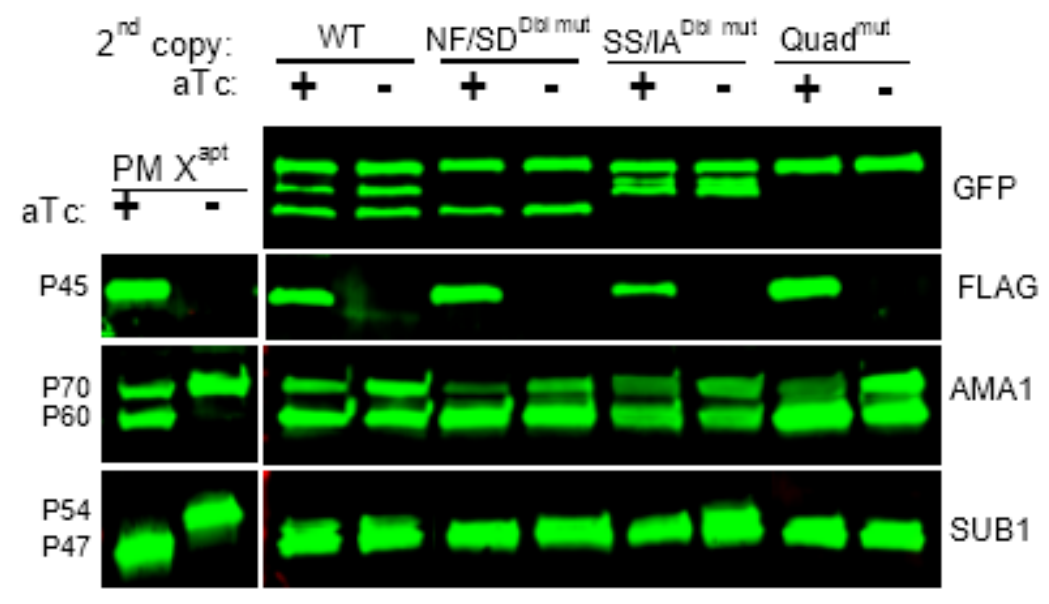

Fig. 2

Figure 2

Autocatalytic processing of PM X is not required for parasite replication and

substrate cleavage. a The parental PM $X^{\text {apt }}$ parasites or PM $X^{\text {apt }}$ parasites expressing the

indicated PM X as a second copy were grown either in the presence or absence of aTc for $96 \mathrm{~h}$.

The starting parasitemia was $1 \%$ across different samples. Shown are the final parasitemia 
percentages after 4 erythrocytic cycles, normalized to that of the PM $\mathrm{X}^{\text {apt }}$ parasites grown in the

presence of aTc for $96 \mathrm{~h}$. Mean values from three independent experiments are shown and

error bars represent standard deviations. $\star \star \star: ~ p<0.001$. $\mathbf{b}$ The parasite lines from a were

MACS synchronized for $3 \mathrm{~h}$ and were grown for the next $45 \mathrm{~h}$ either in the presence or

absence of aTc. Parasites were then harvested and whole cell lysates were prepared. Western blots were performed to detect the expression of the second copy PM X-GFP (top panel), endogenous PM X-FLAG (second panel), AMA1 (third panel) and SUB1 (bottom panel). On the left is the PM Xapt line without second-copy PM X expression. The antibodies used and the specific molecular weights of the different processed forms of proteins are indicated. This experiment was repeated two times and shown is a representative blot.

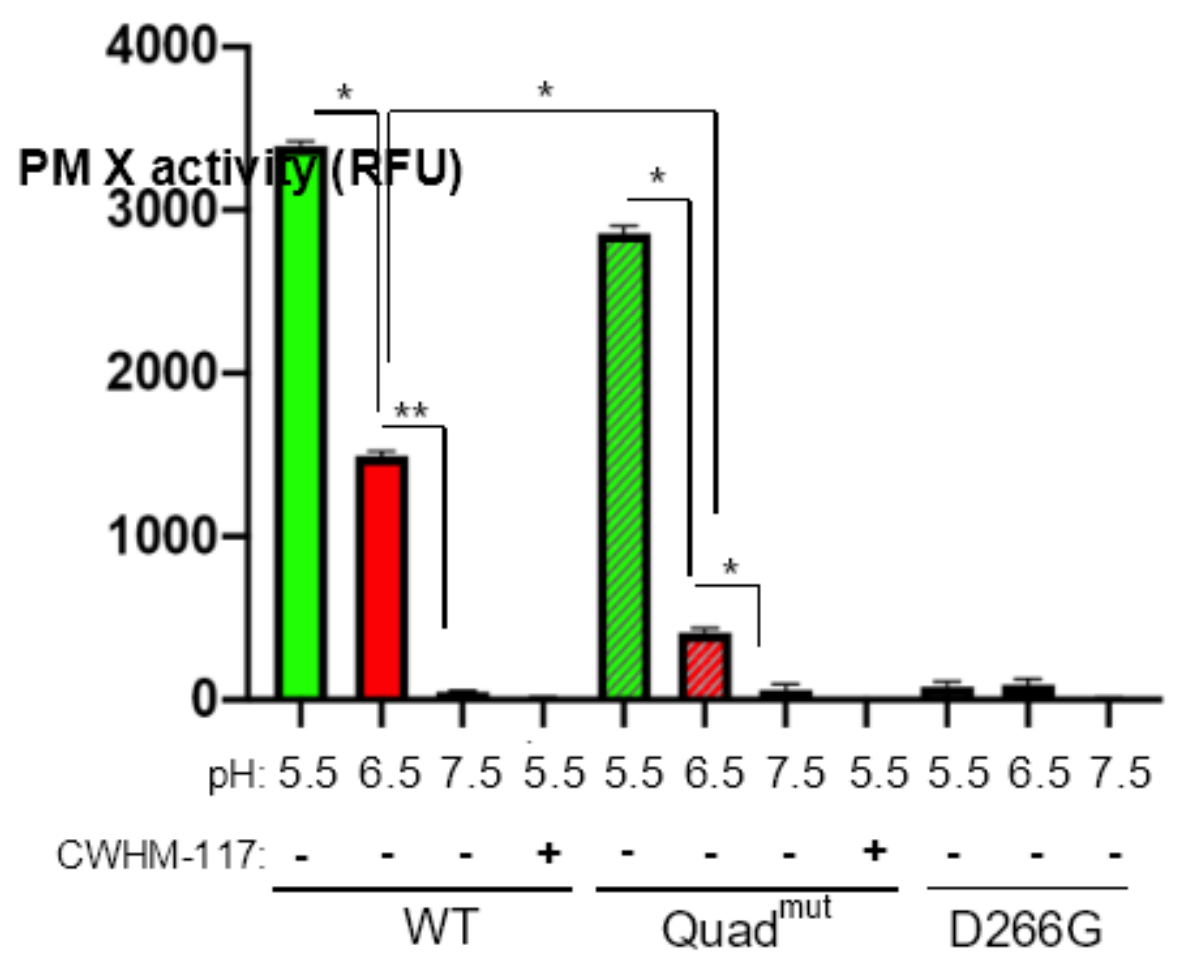

Fig. 3

Figure 3

Proteolytic processing is not necessary for in vitro activity of PM X. Second copy

PM X-GFP was purified from synchronized $42-45 \mathrm{~h}$ schizonts using anti-GFP antibody. The pulled down proteins were then incubated with fluorogenic Rh2N substrate peptide $(1 \mu \mathrm{M})$ at the indicated $\mathrm{pH}$. In control wells, CWHM-117 $(1 \mu \mathrm{M})$ was added to inhibit PM X activity. Reactions were carried out for $1 \mathrm{~h}$ at 
37C. Mean values from three independent experiments are shown and error bars represent standard deviations. $* *$ : $p<0.01, *$ : $p<0.05$.

a

$\mathrm{pH} 8 \mathrm{pH}$ 7 $\mathrm{pH} 5.5$

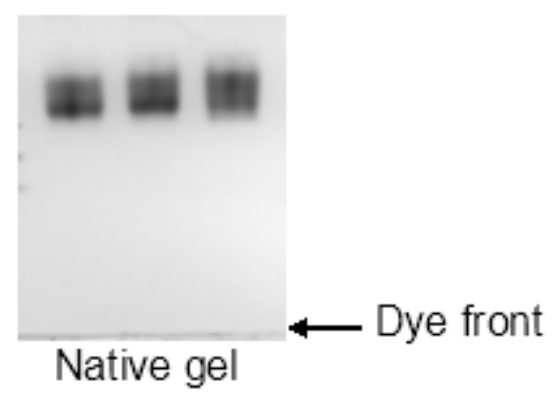

b
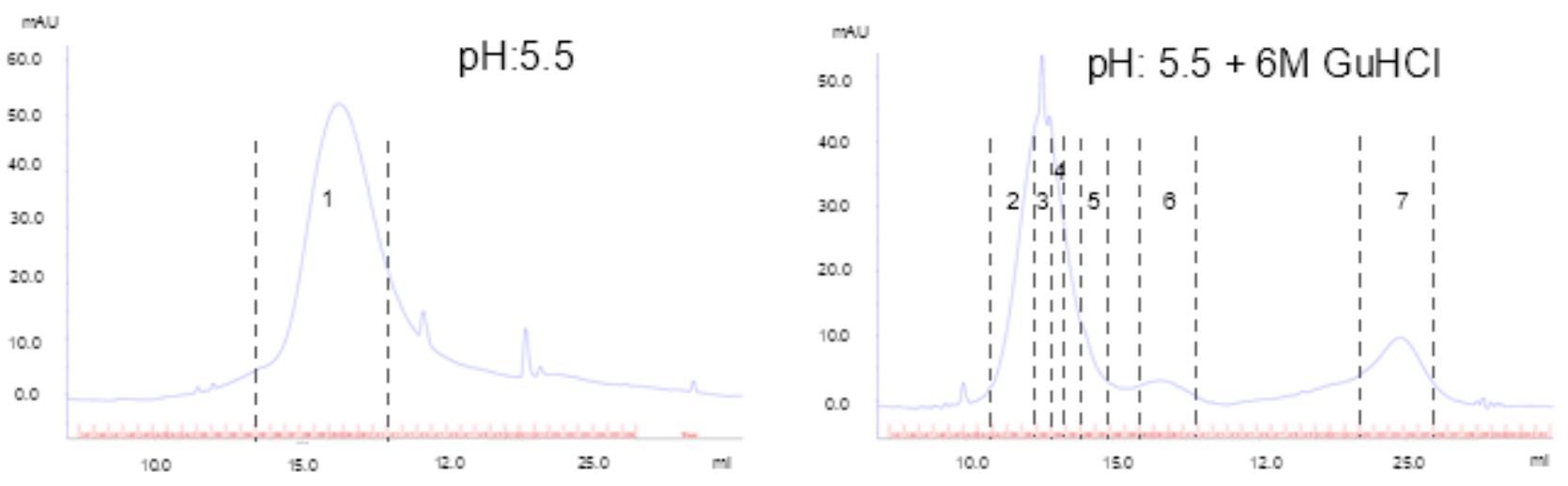

c

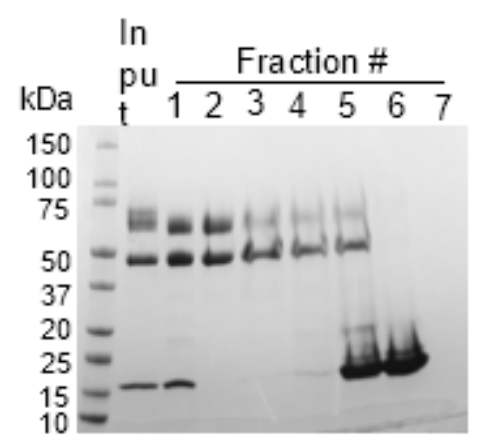

d

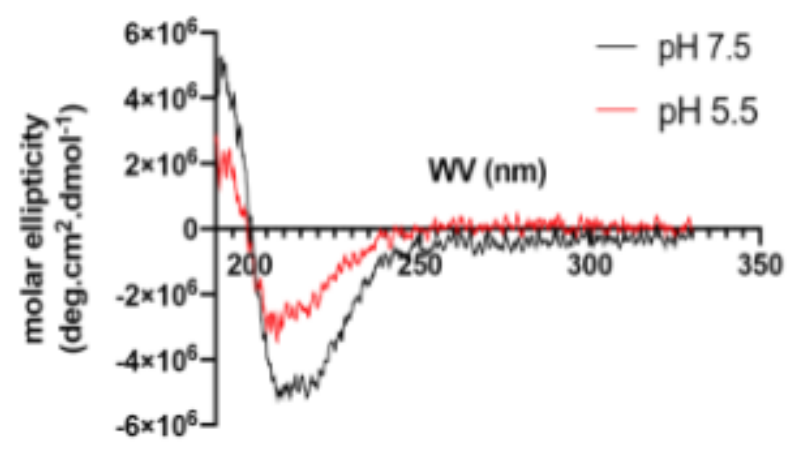

Fig. 4

Figure 4

Acidity-induced conformational changes activate rPM X.

a Aliquots of $\mathrm{rPM} \mathrm{X}$ were preincubated at the indicated $\mathrm{pH}$ and then resolved by PAGE 
under native conditions. The gel was subsequently stained with Coomassie to visualize the protein bands. $\mathbf{b}$ and $\mathbf{c}$ Size-exclusion chromatography of rPM X under acidic conditions ( $\mathrm{pH}$ 5.5, left) or acidic conditions in the presence of $6 \mathrm{M}$ guanidine $\mathrm{HCl}(\mathrm{GuHCl}$, right). Samples were pretreated in the same buffer before loading onto the column. Numbers within dotted lines indicate the fractions that were collected to run on SDS-PAGE followed by Coomassie staining (c). $\mathbf{d}$ Comparison of secondary structures of WT rPM X preincubated at the indicated $\mathrm{pH}$ and analyzed by circular dichroism spectroscopy. Experiment was repeated three times in triplicate. Shown are the mean values from one representative experiment. 
a
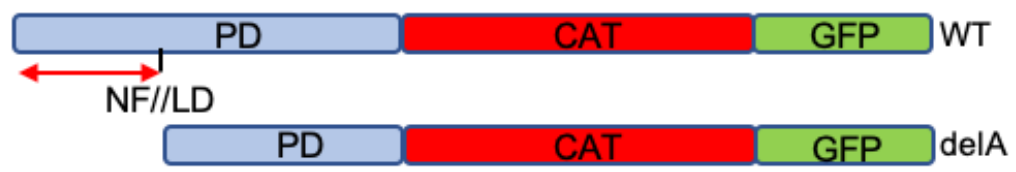

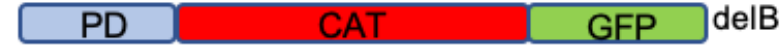

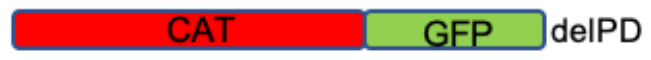

b

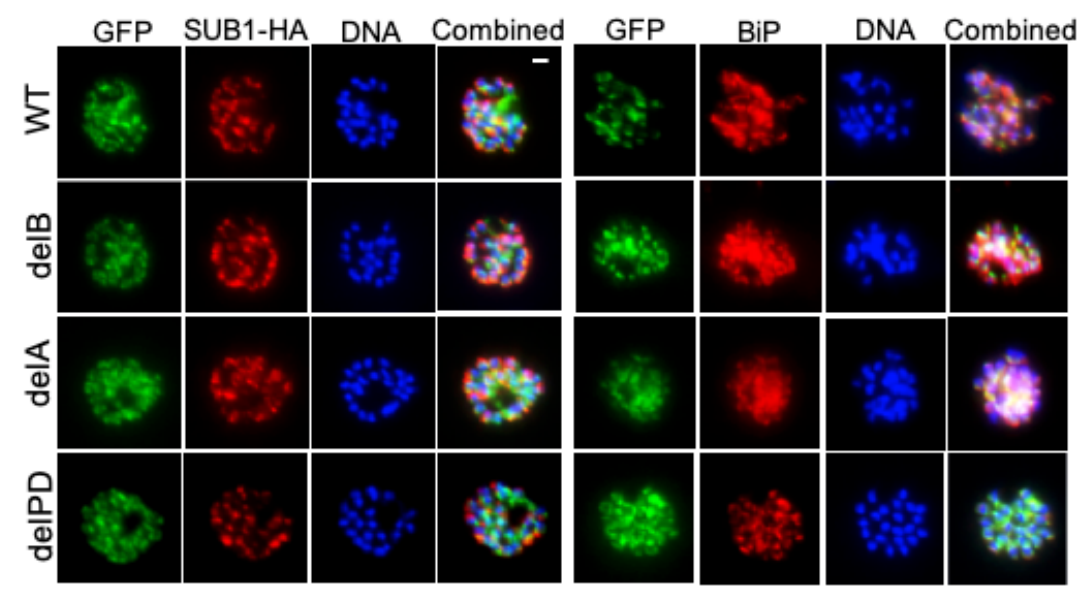

C

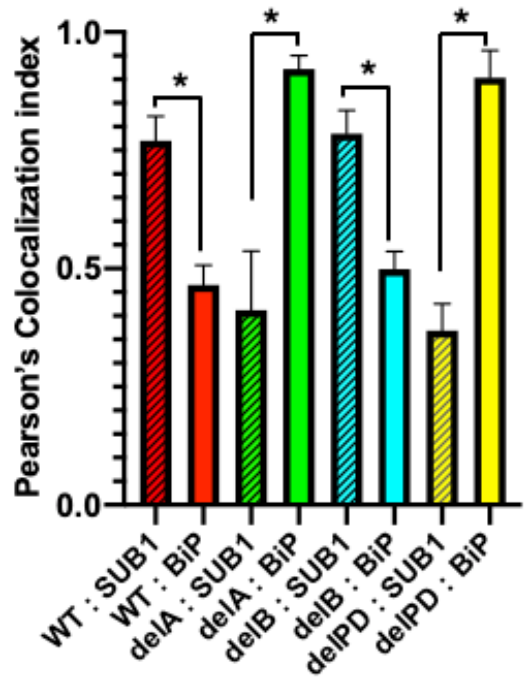

d
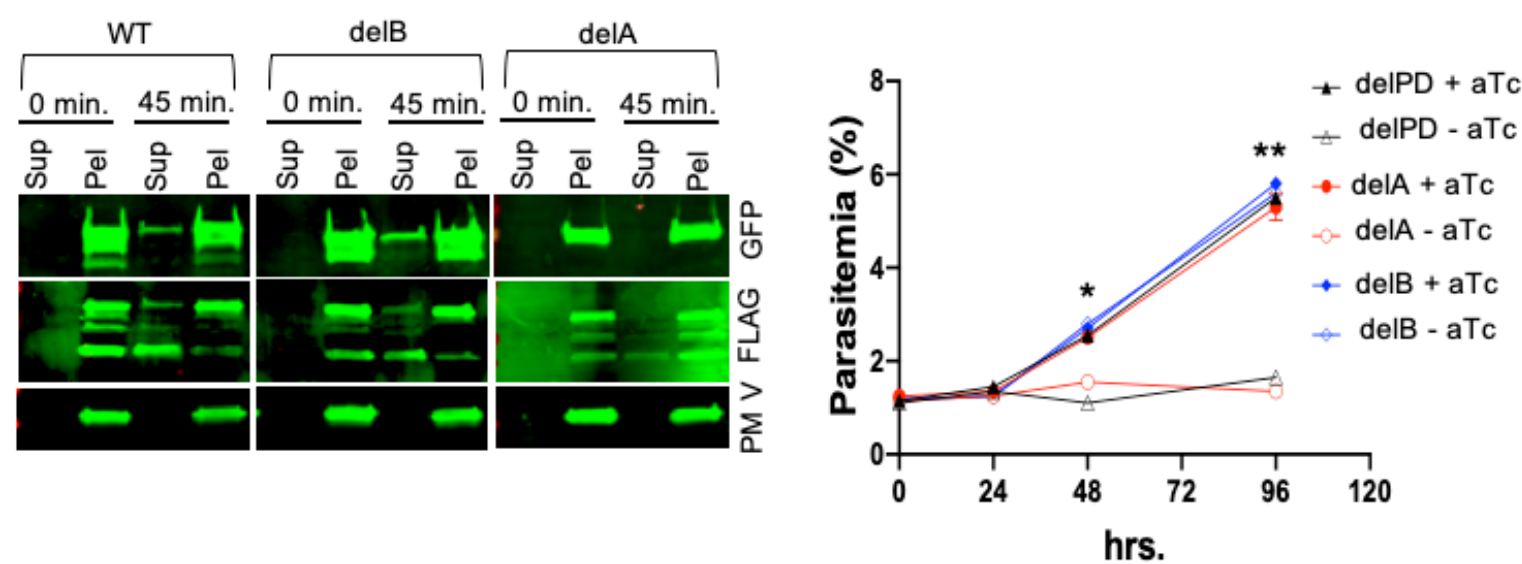

Fig. 5

Figure 5

The N-terminal half of the prodomain (PD) is critical for intracellular trafficking and functionality of PM X. a Scheme of PD truncation constructs that are C-terminally GFP tagged and introduced into the PM $\mathrm{X}^{\text {apt }}$ line as second copies. b Representative IFAs 
showing colocalization between the second copy PM X and either SUB1-3xHA or BiP. Scale

bar: $2 \mu \mathrm{m}$. c Quantification for b. For each line 6 cells were analyzed. Mean values are shown.

Error bars represent standard deviation *: $p<0.05$. $\mathbf{d}$ Zaprinast-induced discharge of second copy

WT and delB but not delA mutant PM X from 41-44 h schizonts. Samples were treated with

$75 \mu \mathrm{M}$ zaprinast for $45 \mathrm{~min}$. Supernatant was then separated from the cellular pellet followed

by western blots with indicated antibodies. Shown are the representative blots from two independent experiments. e Growth curve of indicated parasite lines showing that the delA and delPD mutants failed to rescue the growth defect due to knockdown of PM X. Mean values from three independent experiments are shown and error bars represent standard deviations. ${ }^{* *}: p<0.01, *: p<0.05$.

\section{Figure 6}

PM X is processed in terminal secretory compartments in the parasites. Synchronized 40-43 h schizonts expressing PM X-3xHA were treated separately with the vehicle (DMSO),

E64d $(10 \mu \mathrm{M})$ and $\mathrm{C} 1(1.5 \mu \mathrm{M})$ for $8 \mathrm{~h}$. Samples were fractionated to separate the secreted (supernatant) components from those that remained intracellular (merozoites). PM X-3xHA was pulled down using anti-HA antibody. Western blot was done with both fractions to assess distribution of PM X. Aliquots were blotted for PM V as an intracellular control. Shown are representative blots from two independent experiments.

\section{Figure 7}

PM X cleaves microneme substrates intracellularly, while hoptry substrate cleavage is mediated in a post-secretion manner. $\mathbf{a}$ and $\mathbf{b}$ PM Xapt parasites were grown in presence or absence of aTc to the schizont stage. At $44 \mathrm{~h}$ post invasion, either $\mathrm{C} 1(1.5 \mu \mathrm{M})$ or $\mathrm{E} 64 \mathrm{~d}(10 \mu \mathrm{M})$ was added to the + aTc cultures for $6 \mathrm{~h}$ before harvesting. Samples were blotted assess the cleavage of either AMA1 (a, microneme substrate) or Rh5 (b, rhoptry substrate). Processing of SUB1 and SERA5 were analyzed from the same lysates as indicators of PM X activity and exoneme discharge respectively. Each blot was repeated at least twice.

\section{Figure 8}


PM X and SUB1 partially colocalize with microneme markers (AMA1 and EBA175)

but not with rhoptry neck (RON4) or bulb (RAP1) markers inside the parasites. Synchronized 44-46 h schizonts expressing either PM X-3xHA or SUB1-3xHA were fixed in paraformaldehyde and processed for immunofluorescence assays. After labelling with the indicated antibodies, samples were visualized either by epifluorescence microscopy ( $\mathbf{a}$ and $\mathbf{b}$ ), or by confocal Airyscan microscopy (c). Images in (c) are 2D snapshots. 3D reconstructions are shown in supp fig 6. Scale bars: 2 um. Shown are representative images from two independent experiments.

\section{Figure 9}

A subset of the apically located vesicles in schizonts show colocalization between AMA1 and PM X or SUB1 by immunoEM. Synchronized 44-46 h schizonts expressing either PM X-3xHA (a) or SUB1-3xHA (b) were processed as described in the methods. Thin section samples were labelled for the indicated markers and visualized by immunoelectron microscopy. $18 \mathrm{~nm}$ beads: anti HA antibody, $12 \mathrm{~nm}$ beads: anti AMA1 antibody. Sections showing apical vesicles are magnified from each image. R: rhoptry, N: nucleus. Shown are representative images from two independent experiments.

\section{Supplementary Files}

This is a list of supplementary files associated with this preprint. Click to download.

- Supplementaryfigures.1228.docx

- SupplementaryMSfile1.xls

- SupplementaryMSfile2.xls 